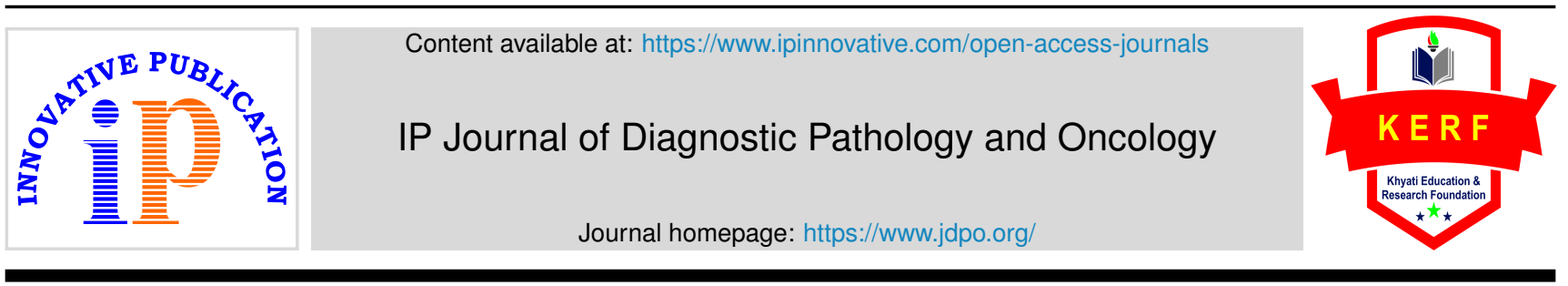

Original Research Article

\title{
A Comparative study for the assessment of diagnostic efficacy and accuracy of various techniques in detection of malarial parasites
}

\author{
Deepika $^{1, *}$, Sathyavathi R Alva ${ }^{2}$ \\ ${ }^{1}$ Dept. of Pathology, Vijayanagar Institute of Medical Sciences (VIMS), Bellary, Karnataka, India \\ ${ }^{2}$ Dept. of Pathology, KVG Medical College and Hospitals, Sullia, Karnataka, India
}

\section{A R T I C L E I N F O}

\section{Article history:}

Received 15-12-2020

Accepted 21-12-2020

Available online 10-03-2021

\section{Keywords:}

Malaria

Peripheral smear

Ag Card test

MPQBC

\begin{abstract}
A B S T R A C T
Background: Malaria is a serious disease caused by Plasmodium species and if left untreated, can be fatal. Four of the known species of Plasmodium commonly infect humans, P. falciparum, P. vivax, P. ovale, and P. malariae.

Due to the serious nature of P. falciparum infections, prompt and accurate diagnosis of the condition is essential for effective management. Keeping in mind the seriousness of the condition and the current availability of diagnostic facilities across India, we decided to compare the efficacy and accuracy of thick and thin peripheral blood smears [which is the gold standard for the diagnosis of malaria in endemic countries] with QBC (Quantitative Buffy Coat) by fluorescent microscopy and Antigen Card test for the detection of malarial parasites.

Aim: 1. To detect the diagnostic accuracy of four different tests in the diagnosis of Malaria (thick and thin peripheral blood smears, QBC and Ag Card test). 2. To evaluate the efficiency of individual tests. 3 . Assessment on comparison of the various techniques in the detection of the malaria

Settings and Design: Comparative Cross- Sectional Study done from November2017 to August 2019 on 250 patients.

Materials and Methods: The patients presenting symptoms suggestive of malaria were included. The total sample size was 250. The blood sample (containing EDTA) was first used to make peripheral blood smears and do Ag card test. The rest of the blood sample was used for QBC.

Statistical Analysis used: The sensitivity, specificity, positive predictive value and negative predictive value will be calculated.

Results: The sensitivity, specificity and accuracy of MPQBC method was the highest among other tests in the present study and it was statistically significant $(\mathrm{p}<0.05)$.

Conclusions: MPQBC (Malarial Parasite Quantitative Buffy Coat) method is superior to other tests. It has good sensitivity and specificity and it can detect low level of parasitemia. It is the most accurate and efficient method for the detection of malarial parasites.
\end{abstract}

(C) This is an open access article distributed under the terms of the Creative Commons Attribution License (https://creativecommons.org/licenses/by/4.0/) which permits unrestricted use, distribution, and reproduction in any medium, provided the original author and source are credited.

\section{Introduction}

According to the latest World Malaria report, released on 30 November 2020, there were 229 million cases of malaria in 2019 compared to 228 million cases in 2018. The estimated number of malaria deaths stood at 409000 in 2019, compared with 411000 deaths in $2018 .^{1-3}$

\footnotetext{
* Corresponding author.

E-mail address: deepikakmr60@gmail.com (Deepika).
}

Malaria is a serious disease caused by the protozoal parasite Plasmodium species, and if left untreated, can be fatal. ${ }^{1}$ Due to the serious nature of $\mathrm{P}$. falciparum infections, prompt and accurate diagnosis of the condition is essential for effective management. ${ }^{4}$

Keeping in mind the seriousness of the condition and the current availability of diagnostic facilities across India, we decided to compare the efficacy and accuracy of thick and thin peripheral blood smears [which is the gold standard for 
the diagnosis of malaria in endemic countries] with QBC (Quantitative Buffy Coat) by fluorescent microscopy and Antigen Card test for the detection of malarial parasites.

\section{Materials and Methods}

\subsection{Inclusion criteria}

Patients of all ages with clinical signs and symptoms of Malaria.

\subsection{Exclusion criteria}

1. Haemolysed or clotted sample.

2. Sample with microbial contamination.

\subsection{Thick and Thin Peripheral blood smear}

These were prepared as per the standard method described elsewhere. The slides were examined by an experienced Pathologist/Microbiologist. Thick smears were reported negative after examination of 20-30 oil immersion fields when no parasites were observed; a thin smear was given negative when no parasites were observed in 20 oil immersion fields. ${ }^{5,6}$

\subsection{Quantitative buffy coat}

Specially designed microhematocrit tubes coated with acridine orange were used. Approximately, 55-60 $\mu \mathrm{l}$ of blood was loaded into the tubes and stopper and float were applied at either ends; the tubes were centrifuged at 12000 RPM in a pre-programmed centrifuge as per the manufacturer's instructions. . ${ }^{5,7}$ The interpretation was done using a standard microscope fitted with Para Lens ultraviolet microscope adaptor and a $\times 60$ objective connected to fiber optic ultraviolet light module. The parasites were seen in buffy coat layer and the interface between RBC and WBC regions. Reports were given as,,,++++++++++ for 0 1, 1-10, 10-100 and more than 100 parasites/QBC fields respectively. ${ }^{3,5,8}$

\subsection{Antigen card test}

Whole blood was collected in a clean container (containing EDTA). Commercially available antigen detection kit used. 4ul whole blood was taken using the sample dropper. The blood sample was added onto the sample pad in the sample well.3 drops of the assay buffer was added in the buffer well. The results were read after 20 minutes. ${ }^{4,9}$ InterpretationPositive-Appearance of the three purplish pink colored lines one each in Pf. Region $(\mathrm{F})$, Pan region $(\mathrm{P})$ and Control region $(\mathrm{C})$ indicated that the sample is reactive for P. falciparum and/or P.vivax/P.malariae/P.ovale only. Negative- Appearance of only one purplish pink coloured line at control ' $C$ ' region Invalid- if no line appeared. ${ }^{4,5}$

\section{Results}

Out of 250 subjects, 150 were found to be positive by Thick peripheral blood smear. The rate of positivity and negativity was $60 \%$ and $40 \%$ respectively. In case of thin peripheral blood smear, 144 were positive and 106 were negative. The rate of positivity and negativity was $57.6 \%$ and $42.4 \%$ respectively. In Antigen Card Test, 155 were positive and 95 were negative with rate of positivity and negativity being $62 \%$ and $38 \%$. In MPQBC, 162 subjects were found to be positive and 88 were negative. The rate of positivity and negativity was $64.8 \%$ and $35.2 \%$ respectively. (Table 1 )

Considering Thick PS as gold standard, the sensitivity, specificity and accuracy of Thin PS was 92.5\%, 92.3\% and 92.4\% respectively. The sensitivity and specificity of $\mathrm{Ag}$ Card Test was $95.5 \%, 94.6 \%$ and $95.2 \%$. The sensitivity, specificity and accuracy of MPQBC was found to be the highest among all i.e $98.7 \%, 97.7 \%$ and $98.4 \%$ respectively. The sensitivity and specificity of Thin PS was found to be the lowest.(Table 2)

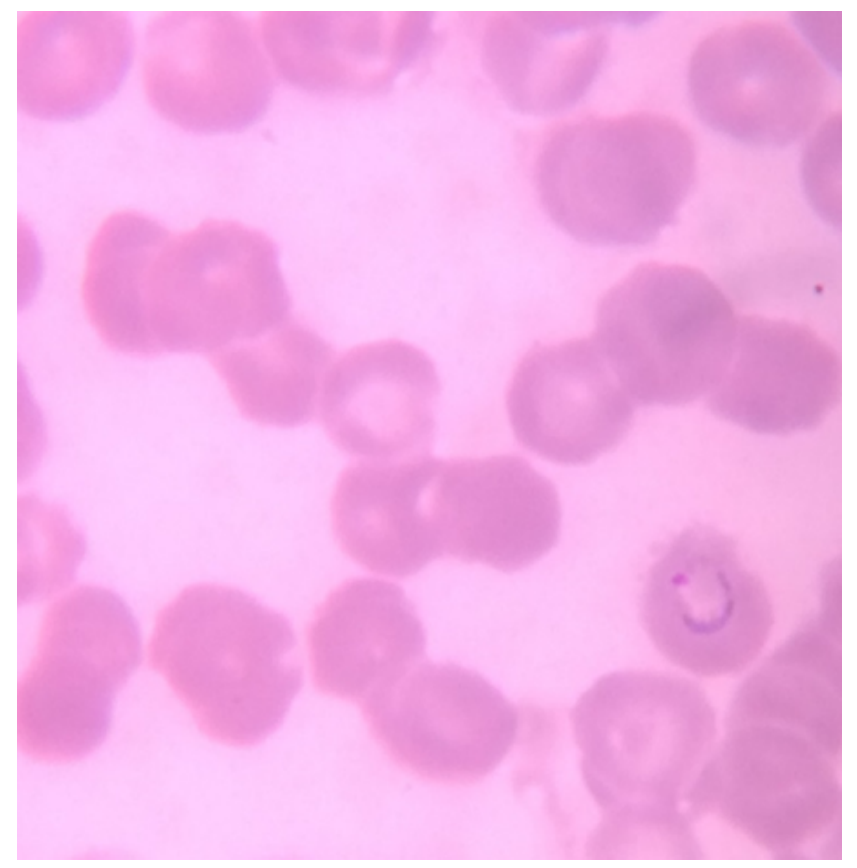

Fig. 1: Ring-formtrophozoite of P.vivax in a thin blood smear.

\section{Discussion}

In many parts of the world, physicians often go with presumptive malaria diagnosis based on clinical symptoms and signs. Various clinical algorithms have been suggested and the best of them predict only up to $50 \%$ of true malaria cases. This method has poor specificity and positive predictive value. It does not allow differentiation of different species of malaria infection. ${ }^{10-12}$ 
Table 1: Rate of detection of malaria by different methods

\begin{tabular}{lcc}
\hline Tests & Results \\
& Positive & Negative \\
Thick Peripheral Smear $(\mathrm{n}=250)$ & $150(60)$ & $100(40)$ \\
Thin Peripheral Smear $(\mathrm{n}=250)$ & $144(57.6)$ & $106(42.4)$ \\
Antigen Card Test $(\mathrm{n}=250)$ & $155(62)$ & $95(38)$ \\
MPQBC $(\mathrm{n}=250)$ & $162(64.8)$ & $88(35.2)$
\end{tabular}

Table 2: Comparison of Sensitivity, Specificity, Positive Predictive Value, Negative Predictive Value and Accuracy of Thin PS, Ag Card Test and MPQBC.

\begin{tabular}{lccccc}
\hline Test & Sensitivity $(\%)$ & Specificity $(\%)$ & $\begin{array}{c}\text { Positive } \\
\text { Predictive Value } \\
(\text { PPV) }(\%)\end{array}$ & $\begin{array}{c}\text { Negative } \\
\text { Predictive Value } \\
\text { (NPV) }(\%)\end{array}$ & Accuracy $(\%)$ \\
Thin PS & 92.5 & 92.3 & 94.4 & 89.7 & 92.4 \\
Ag Card Test & 95.5 & 94.6 & 96.8 & 92.6 & 95.2 \\
MPQBC & 98.7 & 97.7 & 98.8 & 97.7 & 98.4 \\
\hline
\end{tabular}

Table 3: Comparison of Rate of positivity for different methods in various studies.

\begin{tabular}{lcccc}
\hline & Thick PS $(\%)$ & Thin PS(\%) & Ag Card Test $(\%)$ & MPQBC $(\%)$ \\
Our Study & $150(60)$ & $144(57.6)$ & $155(62)$ & $162(64.8)$ \\
Panigrahi K & $62(15.5)$ & $41(10.25)$ & $76(19)$ & $84(21)$ \\
Parija et al & $82(19.95)$ & $45(10.94)$ & $62(15.09)$ & $66(16.06)$ \\
Gulrez et al & $49(61.25)$ & $49(61.25)$ & $71(88.75)$ & $77(96.25)$ \\
\hline
\end{tabular}

Table 4: Sensitivity and specificity of Thin PS, Ag Card Test and MPQBC when compared with Thick PS.

\begin{tabular}{|c|c|c|c|c|c|c|}
\hline & \multicolumn{2}{|c|}{ Thin PS } & \multicolumn{2}{|c|}{ Ag Card Test } & \multicolumn{2}{|c|}{ MPQBC } \\
\hline & $\begin{array}{c}\text { Sensitivity } \\
(\%)\end{array}$ & Specificity (\%) & Sensitivity (\%) & Specificity (\%) & $\begin{array}{c}\text { Sensitivity ( } \\
\%)\end{array}$ & Specificity (\%) \\
\hline Our Study & 92.5 & 92.3 & 95.5 & 94.6 & 98.7 & 97.7 \\
\hline Parija et al & 54.8 & 100 & 75 & 100 & 78.94 & 98 \\
\hline Panigrahi K & 66.12 & 100 & 93.0 & 94.67 & 96.7 & 92.89 \\
\hline
\end{tabular}

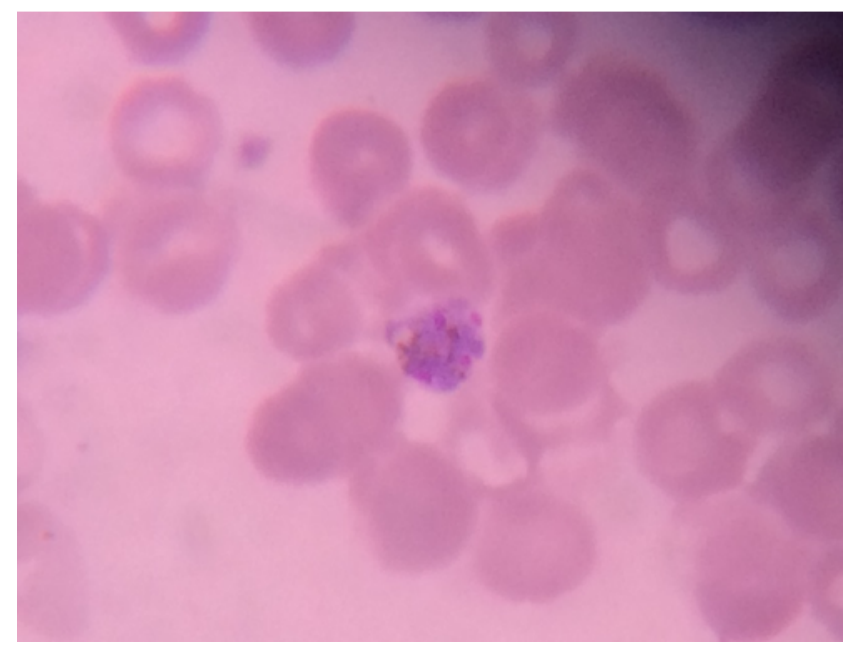

Fig. 2: Schizont of P.vivax in a thin blood smear.

In the present study, we found $150(60.00 \%)$ were positive by microscopic examination of Thick peripheral blood film, 144(57.6\%) were positive by Thin PS, 155(62\%)

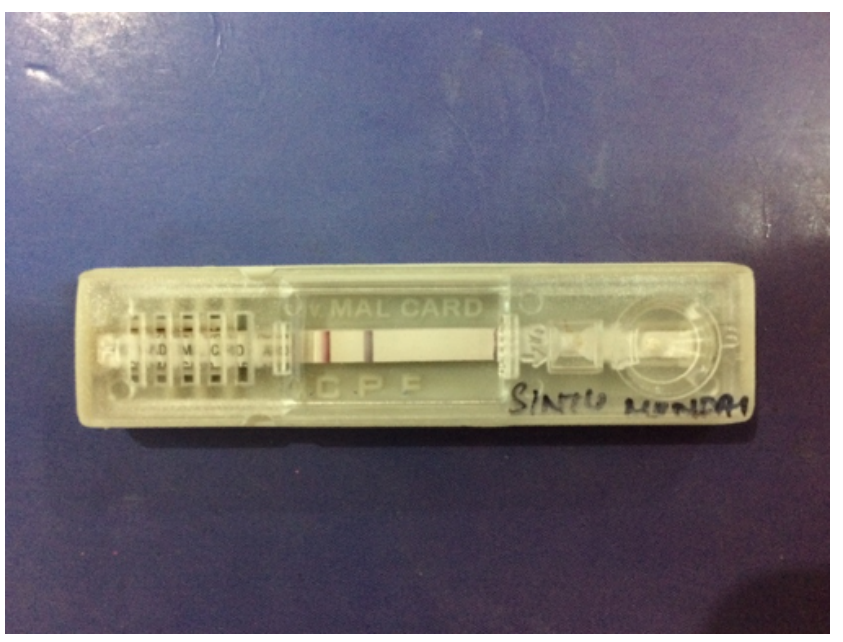

Fig. 3: Positive for Plasmodiumvivax Rapid Visual Antigen test (MAL CARD) 


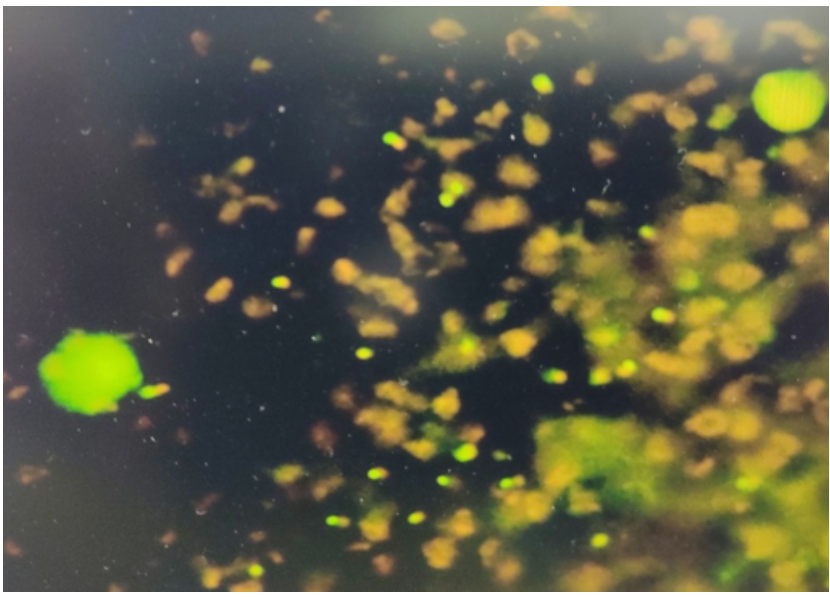

Fig. 4: Trophozoites of P.falciparum stained with Acridine orange (AO) in the QBC UV fluorescence method.

were positive by Ag Card Test and 162(64.8\%) were positive by MPQBC respectively. The rate of positivity was highest with MPQBC method and lowest with Thin PS. The results were similar with a study conducted by Panigrahi et al, ${ }^{10}$ where highest number of cases $84(21 \%)$ were positive by MPQBC method.(Table 3)

In a study by Parija et al, ${ }^{5}$ they found $82(19.95 \%)$ cases were positive by Thick PS examination method which was the highest compared to the other methods, followed by MPQBC which detected 66(16.06\%) cases. Examination of Thin PS has the least rate of positivity of $45(10.94 \%)$; the results were slightly different from our study, where the rate of positivity was highest with MPQBC method.(Table 3)

In another study done by Gulrez et $\mathrm{al},{ }^{4}$ they found $77(96.25 \%)$ cases were positive by MPQBC method, $49(61.25 \%), 48(69.25 \%)$ and $71(8.75 \%)$ were positive by Thick PS, Thin PS and Ag Card Test respectively. This was similar to our study where the rate of positivity was highest with MPQBC method and lowest by Thick PS examination.(Table 3)

In our study, taking Thick peripheral smear as gold standard, we found that thin smear had a sensitivity and specificity of $92.5 \%$ and $92.3 \%$ respectively. The sensitivity and specificity of $\mathrm{Ag}$ Card Test and MPQBC were 95.5\%, $94.6 \%$ and $98.7 \%, 97.7 \%$ respectively.

In a study conducted by Parija et $\mathrm{al}^{5}$ the sensitivity of Thin PS, Ag Card Test and MPQBC were 54.8\%, 75\% and $78.94 \%$ respectively. They found that sensitivity of MPQBC method was highest and Thin PS was the lowest which was similar to the results of our study. They found specificity of Thin PS and Ag Card Test to be $100 \%$ when compared to MPQBC method which was $98 \%$. This result was different from our results where MPQBC method had the highest specificity i.e $97.7 \%$.(Table 4 )

In another study done by Panigrahi $\mathrm{K},{ }^{10}$ the sensitivity of Thin PS, Ag Card Test and MPQBC were $66.12 \%$,
93\% and $96.7 \%$ respectively. They found the sensitivity of MPQBC method to be highest which was similar to our study. However, in their study, the specificity of Thin PS was found to be the highest and lowest for MPQBC method which was different from our study. The dissimilarities were may due to very small number of samples in this group than in our study.(Table 4)

\section{Conclusion}

MPQBC was found to be superior to other tests. It is the most accurate and efficient method for the detection of malarial parasites. Its simple, rapid, reliable, quick and easily mastered method for diagnosis of malaria. It is of much use in laboratories which screen large number of samples and in endemic areas where parasite level is low. It can also be used in highly suspected cases of malaria where parasite could not be detected in peripheral blood film.

In situations where adequate laboratory back up is not available, antigen card test can be employed. However, Leishman stained thin blood smear still appears superior for species identification.

\section{Acknowledgement}

Authors are thankful to Departments of Paediatrics and Medicine of K.V.G Medical College, Sullia D.K for providing institutional support to carry out this study.

\section{Source of Funding}

No financial support was received for the work within this manuscript.

\section{Conflict of Interest}

The authors declare that they have no conflict of interest.

\section{References}

1. World Health Organization. The "World malaria report 2019; 2019. Available from: https://www.who.int/news-room/featurestories/detail/world-malaria-report-2019.

2. Wongsrichanalai C, Wernsdorfer WH, Muth S, Sutamihardja A, Barcus MJ. A Review of Malaria Diagnostic Tools: Microscopy and Rapid Diagnostic Test (RDT). Am J Trop Med Hyg. 2007;77(6):11927. do1:10.4269/ajtmh.2007.77.119.

3. Moody A. Rapid diagnostic tests for malaria parasites. Clin Microbiol Rev. 2002;15(1):66-78.

4. Gulrez M, Varshney KR, Singh YI. Comparative study of various techniques in diagnosis of malaria. Sch J App Med Sci. 2014;2(6C):3060-3.

5. Parija SC, Dhodapkar R, Elangovan S, Chaya DR. A comparative study of blood smear, QBC and antigen detection for diagnosis of malaria. Indian J Pathol Microbiol. 2009;52(2):200-2. 10i:0.4103/0377-4920.48977

6. Berzosa P, de Lucio A, Romay-Barja M, Herrador Z, González $\mathrm{V}$, García L, et al. Comparison of three diagnostic methods (microscopy, RDT, and PCR) for the detection of malaria parasites in representative samples from Equatorial Guinea. Malar $J$.

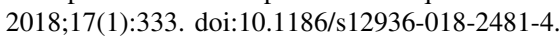


7. World Health Organization. Regional office for South-East Asia. Malaria situation in SEAR countries. India; 2017.

8. Bhandari PL, Raghuveer CV, Rajeev A, Bhandari PD. Comparative study of peripheral blood smear, quantitative buffy coat and modified centrifuged blood smear in malaria diagnosis. Indian J Pathol

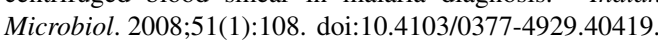

9. Salmani MP, Preeti BM, Peerapur BV. Comparative study of peripheral blood smear and quantitative buffy coat in malaria diagnosis. J Commun Dis. 2011;43(1):57-9.

10. Panigrahi K. A Comparative study of peripheral blood smear, QBC and antigen detectiontest in diagnosis of malaria, in a tertiary care hospital. IJRRMS. 2013;3(3):35-8.

11. Pinto MJ, Rodrigues R, Desouza R, Verenkar MP. Usefulness of quantitative buffy coat blood parasite detection system in diagnosis of malaria. Indian J Med Microbio. 2001;19(4):219.

12. Sreekanth B, Shenoy S, Lella K, Girish N, Reddy R. Evaluation of Blood Smears, Quantitative Buffy Coat and Rapid Diagnostic Tests in the Diagnosis of Malaria. J Bacteriol Parasitol. 2011;02(08):2-2. 100:-10.4172/2155-959/1000125.

\section{Author biography}

Deepika, Senior Resident

Sathyavathi R Alva, Professor and HOD

Cite this article: Deepika, Alva SR. A Comparative study for the assessment of diagnostic efficacy and accuracy of various techniques in detection of malarial parasites. IP J Diagn Pathol Oncol 2021;6(1):1-5. 